\title{
PURIFICATION OF ENDOCRINE DISRUPTER-POLLUTED WATER USING HIGH TEMPERATURE SUPERCONDUCTING HGMS
}

\author{
K. MITSUHASHI ${ }^{\mathrm{a}, *}$, R. YOSHIZAKI ${ }^{\mathrm{a}}, \mathrm{H} \cdot \mathrm{OKADA}^{\mathrm{b}}$, \\ T. OHARA ${ }^{\mathrm{c}}$ and H. WADA ${ }^{\mathrm{c}}$ \\ ${ }^{\mathrm{a}}$ Graduate School of Pure and Applied Sciences, University of Tsukuba, 1-1-1, \\ Tennodai, Tsukuba, Ibaraki 305-8573, Japan; ${ }^{\mathrm{b}}$ Iwate Industrial Promotion Center, \\ 3-35-2, Iiokashinden, Morioka, Iwate 020-0852, Japan; ${ }^{\mathrm{c}}$ National Institute for Materials Science, \\ 3-13, Sakura, Tsukuba, Ibaraki 305-0003, Japan
}

(Received 8 October 2003; Revised 14 November 2003; In final form 20 November 2003)

\begin{abstract}
We have developed a superconducting High Gradient Magnetic Separation (HGMS) system to remove endocrine disrupters from water. The system was able to purify $70 \mathrm{~L}$ of endocrine disrupter-polluted water within about $10 \mathrm{~min}$ without generating any secondary waste. The system consists of pretreatment processes to form magnetized compounds with endocrine disrupters, and an HGMS device to extract them from water by using a strong magnetic force. We produced novel adsorbent made of micron-sized ferric oxide particles that are not only magnetic but also hydrophobic. Because some endocrine disrupters are hydrophobic as well, the ferric oxide particles adsorb endocrine disrupters eluted in water according to hydrophobic interaction. We succeeded in decreasing bisphenol A concentrations in the fortified water from 69 to $16 \mathrm{ng} / \mathrm{L}$ and nonylphenol from 7.0 to $0.9 \mathrm{mg} / \mathrm{L}$. Furthermore, we have successfully demonstrated that we could regenerate the ferric oxide particles, so that they can be re-used for adsorption of endocrine disrupters in water.
\end{abstract}

Keywords: Endocrine disrupter; High gradient magnetic separation; Ferric oxide particle; Hydrophobic magnetite

\section{INTRODUCTION}

The risk of Endocrine-Disrupting Chemicals (hereafter, abbreviated as EDCs) was pointed out in Our Stolen Future [1], which was published in the USA in 1996. EDCs are exogenous chemicals, which influence the normal hormone functions in an organism. Consequently, continuation of the ecosystem becomes questionable when they widely diffuse into the environment [2-7].

*Corresponding author. E-mail: mitsuhashi.Kazunari@nims.go.jp 
In particular, nonyl phenol (hereafter, abbreviated as NP), which is the raw material of a surface active agent, is one of the materials which is recognized as an EDC by the Japanese Ministry of the Environment. Its production is about 17000 tons a year in Japan, and 70000 tons all over the world. Moreover, more than 60 kinds of materials are recognized as a suspicious material besides NP. Bisphenol A (hereafter, abbreviated as BPA), which is one of these suspicious materials, is produced and consumed in large quantities as a raw material of polycarbonate and epoxy resin. Its production is about 350000 tons a year in Japan, and about 1700000 tons all over the world. As described above, EDCs are produced and consumed in large quantities as a raw material of common industrial products, and their evolution and diffusion into the environment are uncontrolled because there are no present regulations.

The most effective method is to discontinue their production thus preventing the diffusion of these materials. However, if their use is prohibited, it may interfere with daily life because important materials in industry use EDCs. Consequently, a massive, high-speed and secure removal of these materials at the origin is required. The main direction of the present research and development is the implementation of the activated charcoal absorption method. However, this may not necessarily be a fundamental solution because the activated carbon used in this method in which the EDCs are adsorbed then becomes a large amount of secondary waste.

The purpose of this study is to develop a new bulk treatment technology, which is applicable to the purification of wastewater containing EDCs and does not produce any secondary waste. It is a technology in which the EDCs are magnetized and purified by a technique called High Gradient Magnetic Separation (hereafter, abbreviated as HGMS). Specifically, EDCs are adsorbed on magnetic particles by their hydrophobic interaction and then magnetically separated from the contaminated water. The features of this method are that the EDCs can be concentrated and extracted from the magnetic particles using an organic solvent, and as a result, the magnetic particles can be recycled.

In this article, the realization of this technology was proved as a result of a massive, high-speed purification experiment using HGMS.

\section{PRINCIPLE AND FEATURES OF MAGNETIC SEPARATION}

The principle of the HGMS is shown in Fig. 1. The magnetic separation is a technique for utilizing magnetic force in order to separate the fine particles, which are dispersed in various dispersion media, such as air, water, seawater, oil, organic solvent, etc. In this study, an HGMS apparatus, which can generate an extraordinary powerful magnetic force, compared to a conventional magnetic separation apparatus, was used. Consequently, it can be applied to various fields because it can effectively exert a magnetic force not only on a ferromagnetic particle but also on paramagnetic and diamagnetic particles.

The advantage of this method is that fine particles trapped by the HGMS apparatus can be desorbed and recycled because the magnetic force can be easily generated and removed (Fig. 2). That is, no secondary waste occurs. Moreover, because the HGMS apparatus has a small space factor for the filter and does not easily clog, it has an advantage that a large amount of separation is possible. 


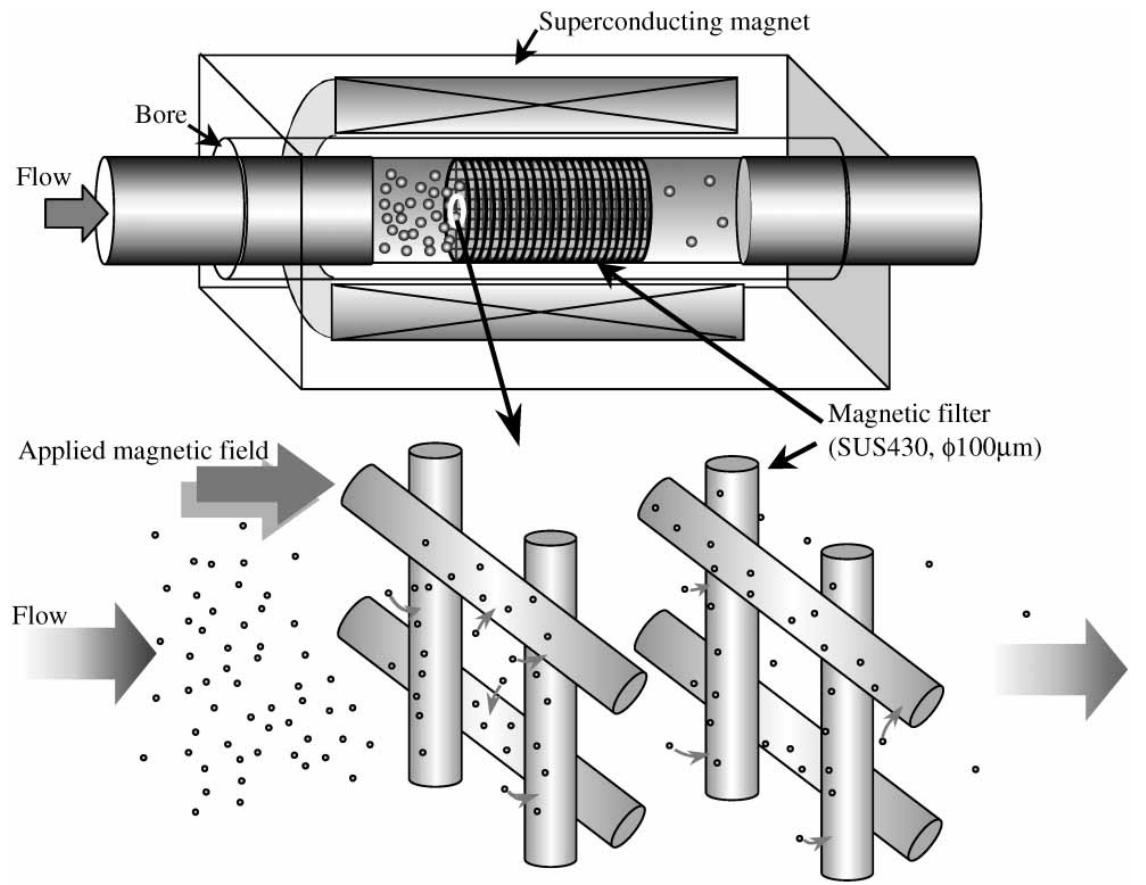

FIGURE 1 Principle of superconducting high gradient magnet separation.

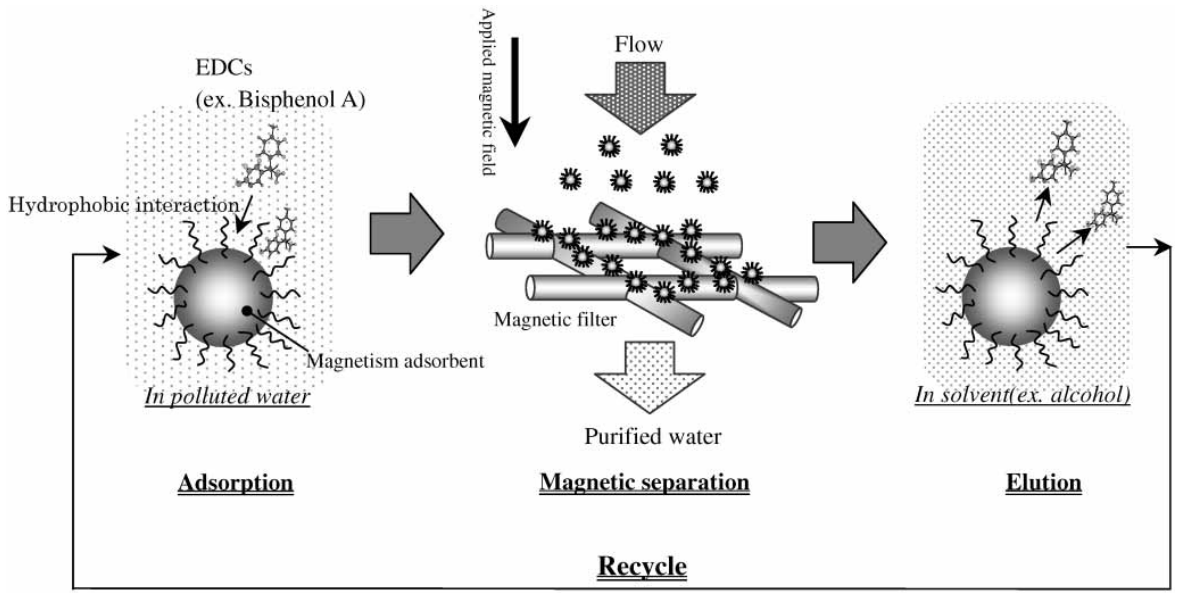

FIGURE 2 Purification cycle of EDCs contaminated water.

\section{EXPERIMENTAL}

\subsection{Synthesis of Magnetic Iron Oxide Fine Particles Modified with Octadecyl Group}

In this experiment, the magnetic iron oxide fine particles were prepared by the ferrite method [25]. The reaction is as follows:

$$
\mathrm{FeSO}_{4}(\mathrm{aq})+\mathrm{Fe}_{2}\left(\mathrm{SO}_{4}\right)_{3}(\mathrm{aq})+8 \mathrm{NaOH}(\mathrm{aq}) \rightarrow \mathrm{Fe}_{3} \mathrm{O}_{4} \downarrow+4 \mathrm{Na}_{2} \mathrm{SO}_{4}(\mathrm{aq})
$$


The feature of this magnetic iron oxide fine particle is that it has a large specific surface area due to the small particle size of tens of $\mathrm{nm}$, and has more hydroxyl groups on the surface of the particle compared to the one prepared by the dry process, which is carried out at a higher temperature. This fine particle is very convenient for the improvement of the immobilization density when the following silane coupling treatment is done.

The silane coupling treatment was carried out according to the following procedures. The fine particles synthesized by the above-mentioned process were dried under reduced pressure, and after the triethyl amine had been added to the dehydrated toluene solvent as a catalyst, $n$-octadecyl tri-chlorosilane was added. The reaction is as follows:

$$
\mathrm{Fe}_{3} \mathrm{O}_{4}-\mathrm{OH}+n-\mathrm{C}_{18} \mathrm{H}_{37} \mathrm{SiCl}_{3} \rightarrow n-\mathrm{C}_{18} \mathrm{H}_{37}-\mathrm{Si}-\mathrm{O}-\mathrm{Fe}_{3} \mathrm{O}_{4}+n \mathrm{HCl}
$$

The n-octadecyl trichlorosilane was purchased from AZMAX, Inc., and the other chemicals were from the Kanto Chemical Co. The dehydrated toluene used in this experiment is for the organic synthesis.

The synthesized product was washed with acetone, and the magnetic iron oxide fine particles modified with octadecylsilane (hereafter, described as $\mathrm{ODS}-\mathrm{Fe}_{3} \mathrm{O}_{4}$ ) were obtained. The existence of the alkyl group in the $\mathrm{ODS}-\mathrm{Fe}_{3} \mathrm{O}_{4}$ was observed by total reflection FT-IR (FTIR-7000T, JASCO Inc.). The absorptions around $1400-1500 \mathrm{~cm}^{-1}$ and $2600-3000 \mathrm{~cm}^{-1}$ in the IR spectrum shown in Fig. 3 indicate its existence.

\subsection{Physical Properties of the Fine Particles}

\subsubsection{Observation with Electron Microscope}

The shape and the size of the fine particles were observed using a transmission electron microscope. The images of the untreated $\mathrm{Fe}_{3} \mathrm{O}_{4}$ and $\mathrm{ODS}-\mathrm{Fe}_{3} \mathrm{O}_{4}$ are shown in Fig. 4. The white bar is $100 \mathrm{~nm}$. The particle size of each sample was from several $\mathrm{nm}$ to several hundred nm. Most of them had a globular shape.

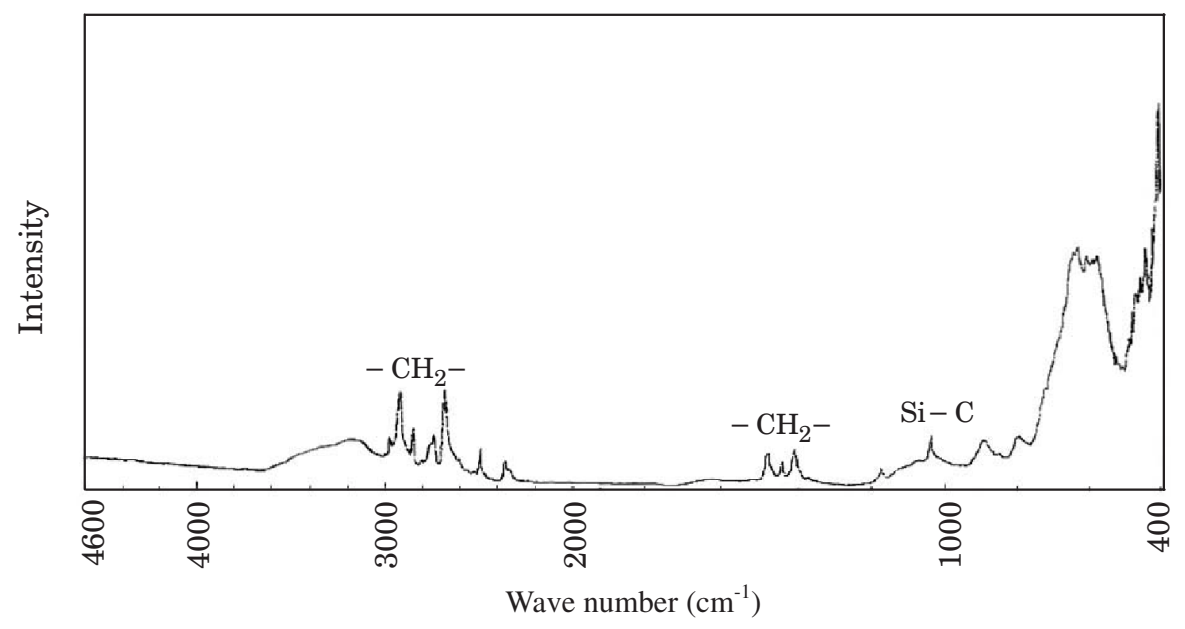

FIGURE 3 IR spectrum of ODS- $\mathrm{Fe}_{3} \mathrm{O}_{4}$. 


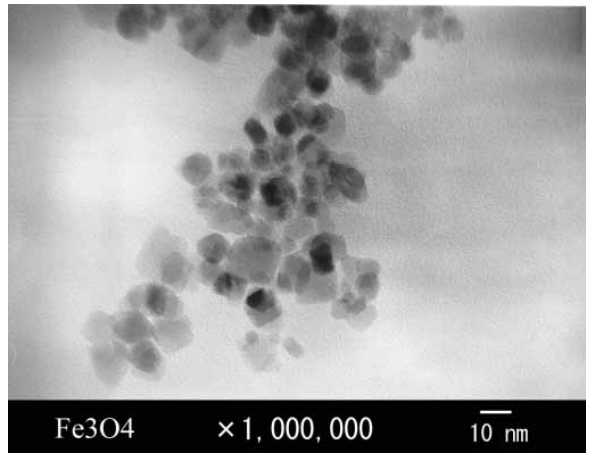

(a)

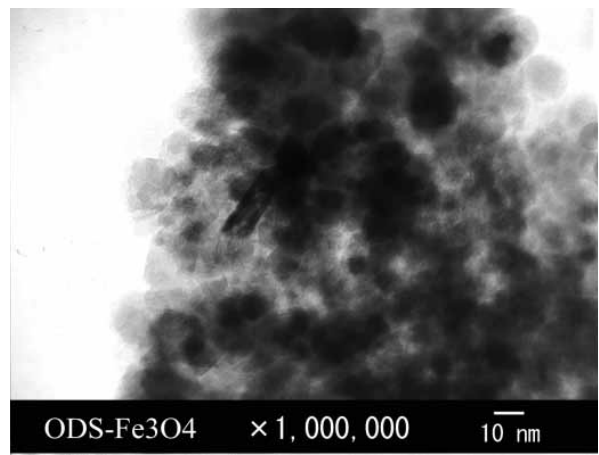

(b)

FIGURE 4 Transmission electron micrography of (a) $\mathrm{Fe}_{3} \mathrm{O}_{4}$ and (b) $\mathrm{ODS}-\mathrm{Fe}_{3} \mathrm{O}_{4}$.

\subsubsection{Measurement of Specific Surface Area}

The specific surface area of the fine particles was measured using a BET full-automatic specific surface area meter (Masorb HM-Model 1201, MOUNTECH Co., Ltd). The specific surface areas of the untreated $\mathrm{Fe}_{3} \mathrm{O}_{4}$ and the ODS- $\mathrm{Fe}_{3} \mathrm{O}_{4}$ were about 70 and $20 \mathrm{~m}^{2} / \mathrm{g}$, respectively. The cause of this decrease in the specific surface area can be attributed to the mutual adhesion of the fine particles during the ODS treatment, or to the decrease in the absorption of nitrogen, which is the measuring gas, due to its hydrophobic property.

\subsubsection{Measurement of Saturation Magnetization}

The saturation magnetization of the fine particle sample was measured using a vibrating sample magnetometer (VSM-5SC-5HF, TOEI INDUSTRY Co., Ltd). The magnetization curve of ODS- $\mathrm{Fe}_{3} \mathrm{O}_{4}$ is shown in Fig. 5. The saturation magnetization of that fine particle sample was $50-60 \mathrm{emu} / \mathrm{g}$, and the residual magnetization was small.

\subsection{Magnetic Separation Apparatus Equipped with Superconducting Magnet}

The magnetic separation apparatus used in this experiment is shown in Fig. 6(a). The flow of water is from a circulating system, which starts from the reservoir and returns to the reservoir after going through the magnetic separation unit. The inside diameter of each pipe is $50 \mathrm{~mm}$.

A high temperature superconducting $\mathrm{Bi}_{2} \mathrm{Sr}_{2} \mathrm{Ca}_{2} \mathrm{Cu}_{3} \mathrm{O}_{x}$ (Bi-2223) magnet [26] is used in the magnetic separation unit. The diameter of the magnetic field space at the centre is $200 \mathrm{~mm}$. The volume of the magnetic field space of $1 \mathrm{~T}$ or more is $11 \mathrm{~L}$ when the maximum magnetic field of $1.7 \mathrm{~T}$ is generated. The three magnetic filters shown in Fig. 6(b) are placed in this space. This magnetic filter consists of an austenitic stainless steel tube with a $50 \mathrm{~mm}$ inside diameter, and 220 sheets of round wire gauze of $50 \mathrm{~mm}$ diameter stacked inside the tube. The diameter of the wire used for the wire gauze is $100 \mu \mathrm{m}$, and the mesh size is 100 . The wire material is a ferrite-type ferromagnetic stainless steel. 


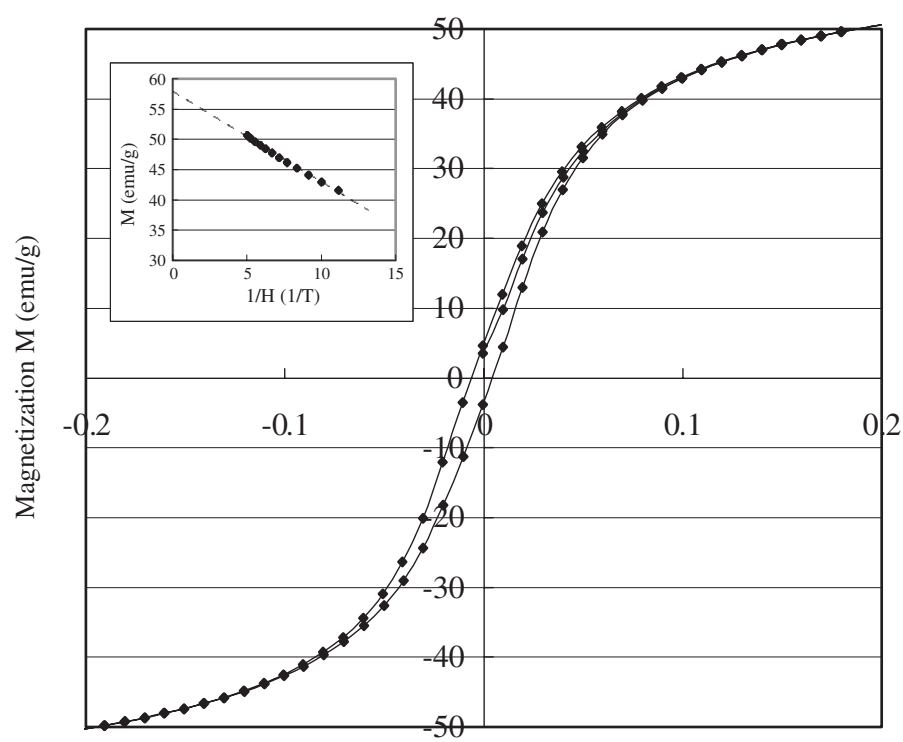

Applied magnetic field $\mathrm{H}(\mathrm{T})$

FIGURE 5 Magnetization curve of ODS- $\mathrm{Fe}_{3} \mathrm{O}_{4}$.

(a)

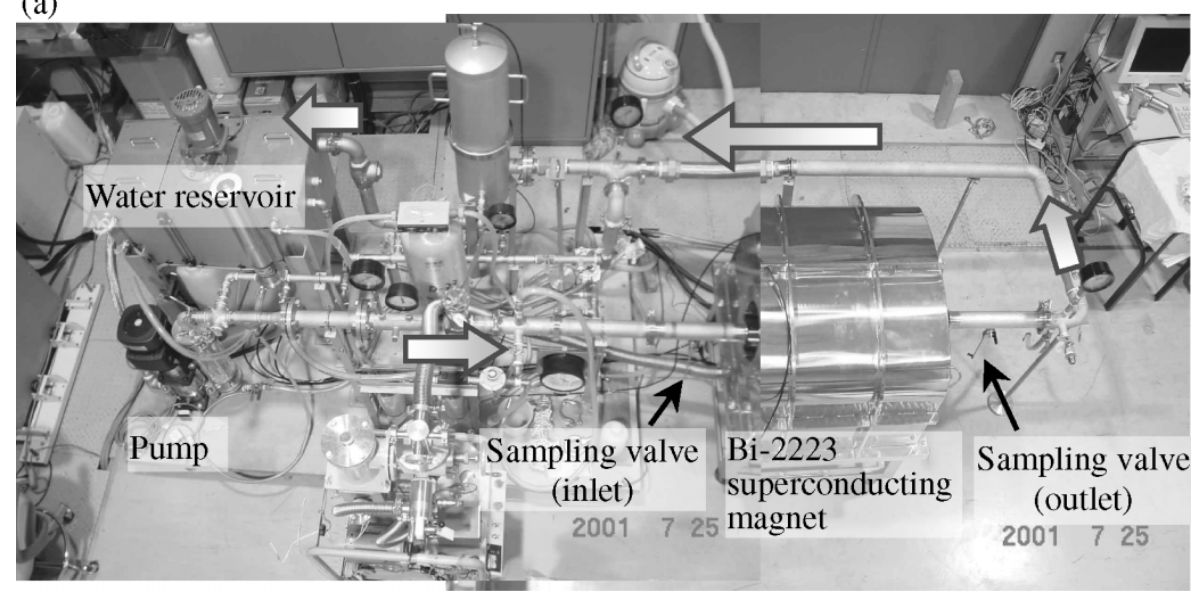

(b)

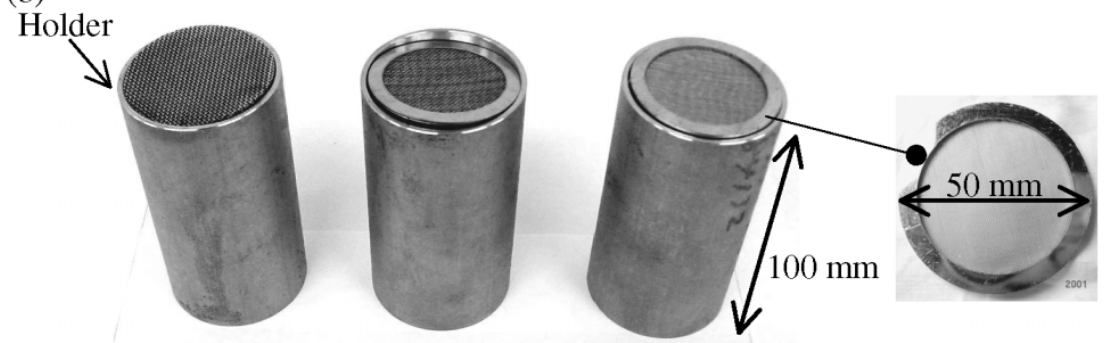

FIGURE 6 (a) HGMS system using high $T_{c}$ superconducting magnet. Large arrow marks represent directions of water flow. (b) Magnetic filters (SUS430, 220 sheets). 


\subsection{Experiment of Magnetic Separation of EDCs}

The experimental conditions were as follows: applied magnetic field: $1.7 \mathrm{~T}$, volume of Mimetic contaminated water: $76 \mathrm{~L}$, flow rate: $20 \mathrm{~L} / \mathrm{min}$, stirring time: $15-20 \mathrm{~min}$, magnetic filter passage time: $10 \mathrm{~min}$. Mimetic contaminated water samples were prepared using BPA and NP, and their concentrations in each sample were adjusted to 69 and $7 \mathrm{mg} / \mathrm{L}$, respectively. The amount of addition of $\mathrm{ODS}-\mathrm{Fe}_{3} \mathrm{O}_{4}$ was 490 and $1100 \mathrm{mg} / \mathrm{L}$ respectively for $76 \mathrm{~L}$ of the mimetic contaminated water. In order to make a homogeneous dispersion of ODS- $\mathrm{Fe}_{3} \mathrm{O}_{4}$, it was dispersed in $100 \mathrm{~mL}$ of ethanol, and then added to the mimetic contaminated water.

The evaluation of the removal efficiency of the EDCs and the iron content was carried out by sampling the mimetic contaminated water in the reservoir and in the pipe before and after passing through the magnetic filter. A high performance liquid chromatograph (LC10ADvp, Shimadzu Corp.) and a ICP emission spectrophotometer (ICPQ-1014, Shimadzu Corp.) were used for analyzing the BPA and NP, and the iron content respectively.

\section{RESULTS AND DISCUSSION}

The changes in the concentration and the removal efficiency of BPA and NP in the reservoir are shown in Fig. 7. When the magnetic separation was completed, the removal efficiency of BPA in the reservoir was about $80 \%$, and that of NP was about $90 \%$. The reason why BPA and NP could be removed is that the hydrophobic interaction worked strongly because the separation was carried out under the reversed phase condition.

It can be understood that the removal efficiency was improved by stirring if the time dependency of the removal efficiency is carefully observed. That is, the removal efficiency of BPA was $50 \%$ immediately after the addition and was $70 \%$ after stirring for $15 \mathrm{~min}$, while the removal efficiency of NP was $80 \%$ after stirring for $20 \mathrm{~min}$. It can be understood that it is important to disperse sufficiently the magnetic fine particles in water by stirring in both cases.

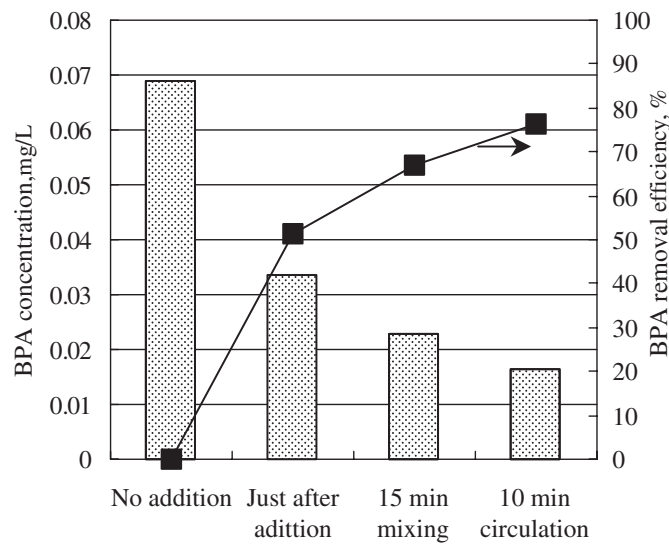

(a)

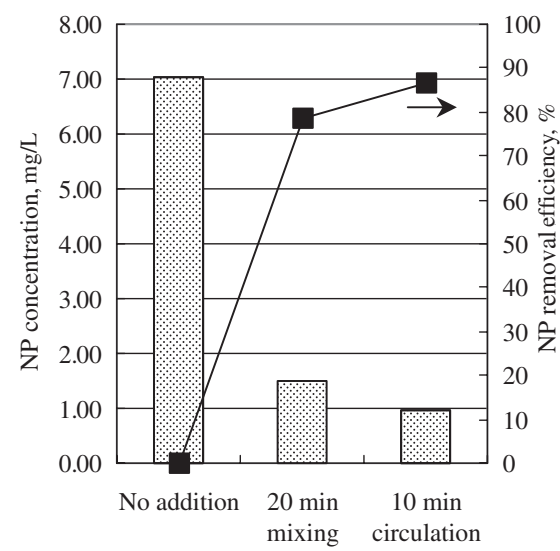

(b)

FIGURE 7 Purification of (a) BPA and (b) NP added water with the HGMS system. 
On the other hand, the extent of the BPA removal was examined when no ODS- $\mathrm{Fe}_{3} \mathrm{O}_{4}$ was added. As a result, the removal efficiency was less than $1 \times 10^{-5} \%$, and $\mathrm{BPA}$ could not be removed at all without the presence of ODS- $\mathrm{Fe}_{3} \mathrm{O}_{4}$. Based on these results, it is clearly understood that BPA is adsorbed on ODS- $\mathrm{Fe}_{3} \mathrm{O}_{4}$, and then desorbed by the magnetic separation.

Figure 8 shows the changes in the ODS- $\mathrm{Fe}_{3} \mathrm{O}_{4}$ concentration when the magnetic separation of BPA was carried out. The ODS- $\mathrm{Fe}_{3} \mathrm{O}_{4}$ concentration at the outlet was always below the lower limit of detection $(1 \mathrm{mg} / \mathrm{kg})$ for $10 \mathrm{~min}$ as shown in the figure. In this case, if the magnetic filter was clogged, it was postulated that a breakthrough occurred and the concentration at the outlet increased. However, the result of the experiment did not support such clogging. Consequently, it can be understood that there was no clogging and there was enough of a margin in the removal capacity.

The discrepancy in the amount of ODS- $\mathrm{Fe}_{3} \mathrm{O}_{4}$ between the amount added to the reservoir and the amount dispersed in the piping was considered. The amount of ODS- $\mathrm{Fe}_{3} \mathrm{O}_{4}$ added to the reservoir was $490 \mathrm{mg} / \mathrm{kg}$, and the concentration at the inlet was $100 \mathrm{mg} / \mathrm{kg}$ at the beginning of the magnetic separation (Fig. 8). This means that about $20 \%$ of ODS- $\mathrm{Fe}_{3} \mathrm{O}_{4}$ added to the reservoir arrived at the inlet of the magnetic separation unit. Due to the precipitate of ODS- $\mathrm{Fe}_{3} \mathrm{O}_{4}$ was observed at the bottom of the reservoir when the experiment was completed, it can be considered that precipitation also occurred in the piping and the reservoir during the experiment. In order to prevent this phenomenon, it is necessary to avoid flocculation of the fine particles and to have sufficient stirring.

The concentration and extraction of $\mathrm{BPA}$ was examined. The $\mathrm{ODS}-\mathrm{Fe}_{3} \mathrm{O}_{4}$ fine particles collected from the magnetic filter were washed with a small amount of ethanol in order to extract the BPA adsorbed on the fine particles. Figure 9 shows the relations between the number of washings of the fine particles and the concentration of eluted BPA. They were washed with $500 \mathrm{~mL}$ of ethanol for the first time and then subsequently with $250 \mathrm{~mL}$ of ethanol for the second time and later. It can be understood from this

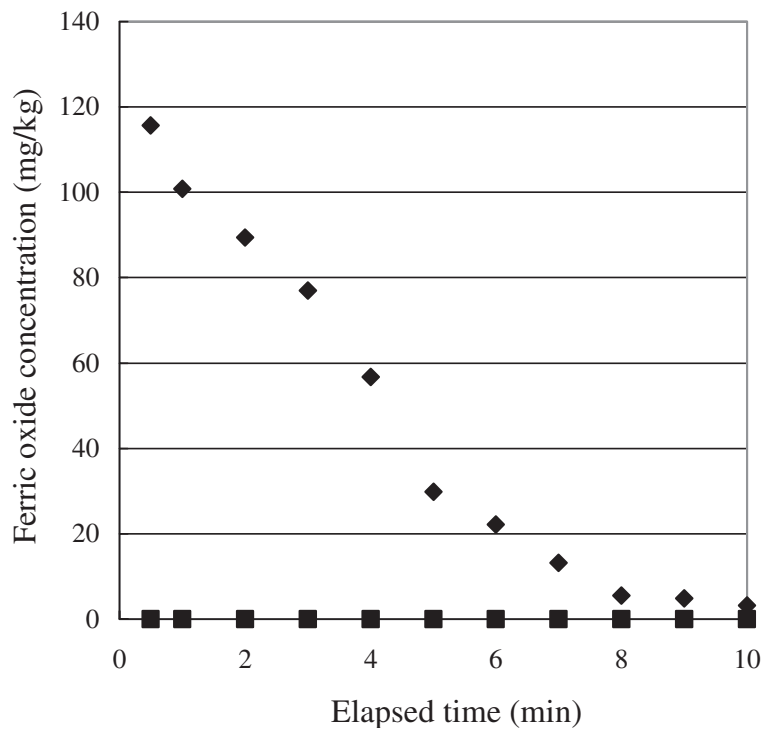

FIGURE 8 Removal of ODS- $\mathrm{Fe}_{3} \mathrm{O}_{4}$ by the HGMS system. inlet; outlet. 


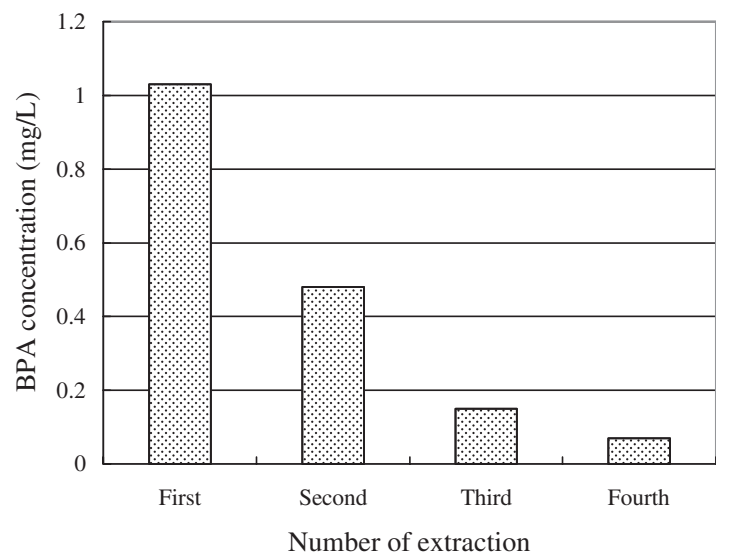

FIGURE 9 Extraction of BPA from recovered ODS- $\mathrm{Fe}_{3} \mathrm{O}_{4}$.

figure that BPA was concentrated to about 10 times the initial concentration by one washing. In this experiment, the amount of collected ODS- $\mathrm{Fe}_{3} \mathrm{O}_{4}$ was about $20 \%$ of the amount added to the reservoir. Therefore, if the fine particles, which were precipitated or adhered in the piping and the reservoir, could be collected, the concentration factor could be further improved.

The following procedures were done in order to collect ODS- $\mathrm{Fe}_{3} \mathrm{O}_{4}$ from the magnetic filter. That is, after the magnetic field was $0 \mathrm{~T}$, and the magnetic force between the filter and the fine particles was removed, water was passed through the filter. Thus, the filter can be easily washed only by removing the magnetic field.

The recovery of the adsorptivity after the washing was also examined. The concentration of eluted BPA decreased by $1 / 2$ whenever the amount of washing cycles was increased. When the adsorption ability of the ODS- $\mathrm{Fe}_{3} \mathrm{O}_{4}$ between the one after washing fourth time and the one immediately after the chemosyntheses was compared, it was almost the same. Consequently, it has been confirmed that it can be recycled.

In the future, the investigations are required concerning the immobilization density of the ODS- $\mathrm{Fe}_{3} \mathrm{O}_{4}$, the EDCs adsorption property, the stirring method, etc. Furthermore, optimization of the system is also required. Moreover, we are going to examine the applicability of this technique in the field such as industrial wastewater, which contains EDCs.

\section{References}

[1] D. Dumanoski, J.P. Myers and T. Colborn, Our Stolen Future, Penguin, New York, 1996.

[2] S. Jobling, T. Reynolds, R. White, M.G. Parker and J.P. Sumpter, A variety of environmentally persistent chemicals, including some phthalate plasticizers, are weakly estrogenic, Environmental Health Perspective 103 (1995), 582.

[3] Preliminary Report on Chemical Industry, The Japanese Ministry of Economy, Trade and Industry, 1995.

[4] R. Gerber and R.R. Birss, High Gradient Magnetic Separation, Research Studies Press, John Wiley \& Sons, Chichester, 1983.

[5] J. Svoboda, Magnetic Methods for the Treatment of Minerals, Elsevier, Amsterdam, 1987.

[6] Patent in Japan. Publication number: 05-112789, 7 May 1993.

[7] T. Ohara, H. Kumakura and H. Wada, Magnetic separation using superconducting magnets, Physica $C$ 357-360 (2001), 1272. 